\title{
Size Effects in Antiferromagnetic NiO Nanoparticles
}

\author{
S. Baran ${ }^{a}$, A. Hoser ${ }^{b}$, B. Penc ${ }^{a}$ And A. Szytula ${ }^{a}$ \\ ${ }^{a}$ Institute of Physics, Jagiellonian University, W.S. Reymonta 4, 30-059 Kraków, Poland \\ ${ }^{b}$ Helmholtz-Zentrum Berlin für Materialen und Energie GmbH, Hahn-Meitner Platz 1, D-14 109 Berlin, Germany
}

(Received April 28, 2014; in final form December 19, 2014)

\begin{abstract}
$\mathrm{X}$-ray and neutron diffraction as well as magnetometric methods were used in order to investigate crystal and magnetic structure together with magnetic properties of nickel oxide $\mathrm{NiO}$ obtained from thermal decomposition of $\mathrm{Ni}(\mathrm{OH})_{2}$. It has been found that crystal unit cell volume and crystal unit cell deformation parameter decrease with increasing decomposition temperature $T_{\mathrm{d}}$ while grain size increases. The results of magnetization, magnetic susceptibility and neutron diffraction measurements reveal a formation of antiferromagnetic order with uncompensated magnetic moment below the Néel temperature. Magnetization together with coercive field decreases with increasing $T_{\mathrm{d}}$. The neutron diffractogram of sample obtained at $240^{\circ} \mathrm{C}$ indicates broadening of both the peaks of nuclear and magnetic origin. The magnetic ordering may be described by a propagation vector $\boldsymbol{k}=\left[\frac{1}{2}, \frac{1}{2}, \frac{1}{2}\right]$.
\end{abstract}

DOI: 10.12693/APhysPolA.129.35

PACS: 61.05.F-, 75.30.Cr, 75.47.Lx, 75.50.Ee

\section{Introduction}

Magnetic properties of nanoparticle systems are subject of continuously growing interest driven by both fundamental research and technological applications [1].

Among number of physical properties especially interesting is an influence of grain size on magnetic ordering. In this work properties of $\mathrm{NiO}$ nanoparticle samples are reported. $\mathrm{NiO}$ crystallizes in the cubic $\mathrm{NaCl}$-type structure and is antiferromagnetic below $520 \mathrm{~K}[2]$. Neutron diffraction investigations indicate that its magnetic order is described by the propagation vector $\boldsymbol{k}=\left[\frac{1}{2}, \frac{1}{2}, \frac{1}{2}\right]$. The Ni magnetic moments are arranged in ferromagnetic sheets parallel to the (111) plane with magnetic moments in neighbouring planes being antiparallel. The moments lie within the (111) plane [3-5]. NiO can also be obtained as nanoparticles. Magnetic properties of such samples are being intensively investigated. The works $[6-8]$ report different properties: superparamagnetism, spin glass behavior or complex antiferromagnetic core with uncompensated magnetic moment and disordered surface shell. All these properties result from macroscopic magnetic measurements.

There are no papers that would report on correlation between grain size and crystal structure. Also the data on grain size influence on microscopic parameters of magnetic structure, the value and direction of magnetic moment are missing.

In this work we report the results of X-ray and neutron diffraction and magnetometric measurements of $\mathrm{NiO}$ samples obtained by dehydration of hydrooxide $\mathrm{Ni}(\mathrm{OH})_{2}$ at different temperatures. From these data the influence of nanoparticle size on physical properties of $\mathrm{NiO}$ is deduced.

\section{Experiment}

The samples of $\mathrm{NiO}$ were synthesized by dehydration of $\mathrm{Ni}(\mathrm{OH})_{2}$ hydrooxide in air for $3 \mathrm{~h}$ at various tempera- tures: $240,290,350,400,470,600$, and $1300^{\circ} \mathrm{C}$. The obtained samples were checked by X-ray diffraction $\left(\mathrm{Cu} K_{\alpha}\right.$ radiation) at room temperature with the use of a Philips PANalytical X'PERT Pro-type diffractometer.

DC magnetic measurements were carried out using a vibrating sample magnetometer (VSM) option of the Quantum Design PPMS platform. Two types magnetic measurements were performed: collecting ZFC (zero field cooling) and FC (field cooling) magnetic susceptibility curves at applied field of $H=50$ Oe from 1.9 and $350 \mathrm{~K}$ and measuring magnetization curves up to $90 \mathrm{kOe}$ at $2 \mathrm{~K}$.

Neutron diffraction patterns were recorded with the use of the E6 diffractometer located at the Berlin Neutron Scattering Center in the Helmholtz Center Berlin for Materials and Energy. The incident neutron wavelength was $2.447 \AA$. The data were collected at several temperatures between 2.6 and $556 \mathrm{~K}$.

The Rietveld-type program FullProf [9] was used for $\mathrm{X}$-ray and neutron diffraction data analysis.

\section{Results}

\subsection{Crystal structure from X-ray data}

The results of the X-ray and neutron diffraction indicate that the samples are single-phased with a cubic crystal structure of the NaCl-type.

In Fig. 1 the X-ray diffraction patterns of the samples annealed at $1300^{\circ} \mathrm{C}$ and $240{ }^{\circ} \mathrm{C}$ are presented in a wide $2 \theta$ range. Figure 2 shows diffraction data for all the samples in limited $2 \theta$ range between $30^{\circ}$ and $50^{\circ}$, containing (111) and (200) Bragg reflections. With increase of annealing temperature a gradual decrease of FWHM (full width at half maximum) parameter is observed. For numerical analysis of the Bragg reflection broadening the modified Debye-Scherrer formula [10] was adopted

$$
\frac{H_{h k l} \cos \theta_{h k l}}{K \lambda}=\frac{1}{D_{h k l}}+\left\langle\frac{\Delta a}{a}\right\rangle \frac{4}{K \lambda} \sin \theta_{h k l}
$$

where $K$ is a Scherrer constant, $\lambda-\mathrm{X}$-ray wavelength, 

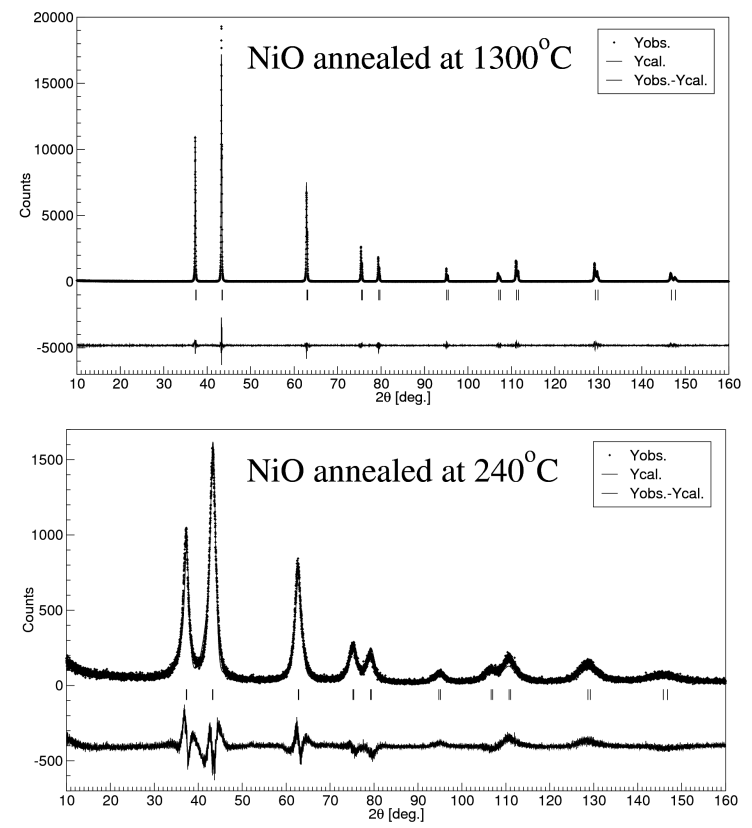

Fig. 1. X-ray diffraction patterns of $\mathrm{NiO}$ samples annealed at $1300{ }^{\circ} \mathrm{C}$ and $240{ }^{\circ} \mathrm{C}$.

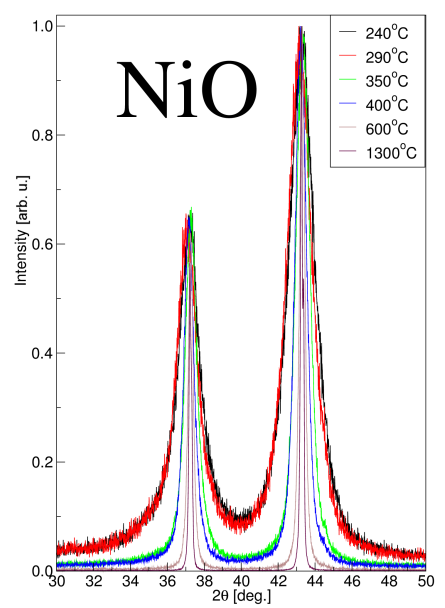

Fig. 2. X-ray diffraction patterns of $\mathrm{NiO}$ samples annealed at different temperatures. Only $2 \theta$ range from $30^{\circ}$ to $50^{\circ}$ is shown in order focus in detail on the (111) and (200) Bragg reflections. For better visibility the reflection height is normalized with respect to that of (200).

TABLE I

Structural parameters of $\mathrm{NiO}$ nanosamples determined from the X-ray data at room temperature: $T_{\mathrm{d}}-$ dehydration temperature, a - lattice parameter, D - grain size, $\frac{\Delta a}{a}$ - deformation parameter of unit cell.

\begin{tabular}{c|c|c|c}
\hline \hline$T_{\mathrm{d}}\left[{ }^{\circ} \mathrm{C}\right]$ & $\mathrm{a}[\AA]$ & $\mathrm{D}[\AA]$ & $\left\langle\frac{\Delta a}{a}\right\rangle$ \\
\hline 240 & $4.1827(2)$ & $80(2)$ & $0.55(20)$ \\
290 & $4.1839(2)$ & $88(3)$ & $0.14(2)$ \\
350 & $4.1799(3)$ & $217(5)$ & $0.06(2)$ \\
400 & $4.1789(3)$ & $274(7)$ & $0.05(2)$ \\
600 & $4.1770(2)$ & $594(17)$ & $0.04(1)$ \\
1300 & $4.1766(1)$ & $2557(135)$ & $0.02(3)$
\end{tabular}

$H_{h k l}$ is a full-width at half-height of the Bragg reflection, $a$ is a lattice constant, while $D_{h k l}$ and $\left\langle\frac{\Delta a}{a}\right\rangle$ refer to grain size and deformation parameter, respectively. The determined values of $D_{h k l}$ and $\left\langle\frac{\Delta a}{a}\right\rangle$ are listed in Table I. One can notice that both the lattice constant $a$ and $\left\langle\frac{\Delta a}{a}\right\rangle$ parameter decreases while $D_{h k l}$ increases with increasing synthesis temperature. The determined values of grain sizes are close to those reported in Ref. [6].

\subsection{Magnetic data}

Figure 3 shows temperature dependence of ZFC and FC dc magnetic susceptibility for samples synthesized at different temperatures. Analysis of these data leads to the following conclusions:

1. Magnetic susceptibility decreases with increase of dehydration temperature. Similar dependence is reported in Ref. [6].

2. Difference between ZFC and FC curves is observed. The difference is large for sample synthesized at low temperatures and decreases with increasing dehydration temperature $T_{\mathrm{d}}$.

3. Magnetic susceptibility $\mathrm{ZFC}$ curves for samples synthesized at 240 and $290^{\circ} \mathrm{C}$ shows a broad maximum near $220 \mathrm{~K}$.

4. For all the samples a maximum in ZFC curve associated with a drop decrease of FC curve is observed at low temperatures near $10 \mathrm{~K}$. Similar effect is observed for the $\mathrm{NiO}$ nano sample with grain size equal to $30 \AA$ (see Fig. 3 in Ref. [7]).

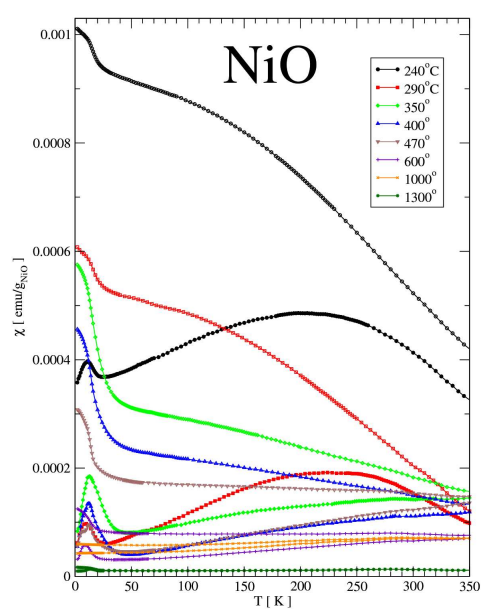

Fig. 3. Temperature dependence of dc magnetic susceptibilities: ZFC and FC curves for the samples synthesized at different temperatures. The open symbols refer to ZFC curves while the solid ones to FC curves.

Figure 4 shows hysteresis loop curves collected at $T=$ $2 \mathrm{~K}$ for $\mathrm{NiO}$ samples synthesized at different temperatures. The following conclusions can be drawn from the data: 
(a)
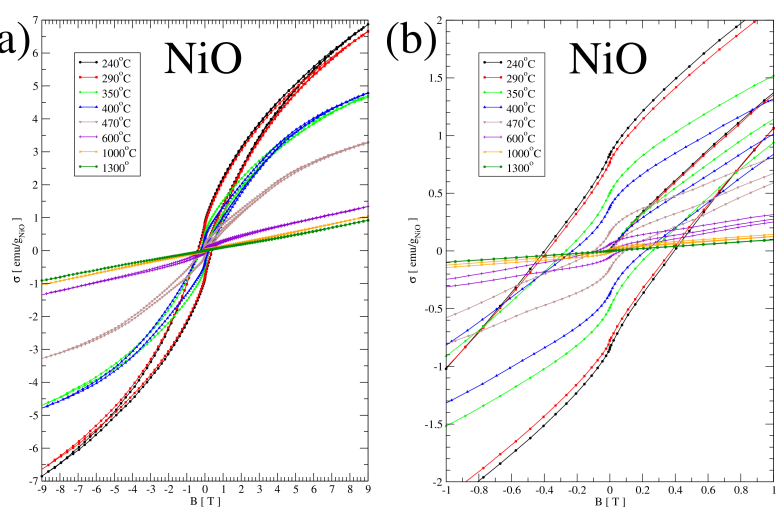

Fig. 4. Isothermal magnetization of $\mathrm{NiO}$ samples vs. external magnetic field, measured at $2 \mathrm{~K}$ : (a) data up to $9 \mathrm{~T}$, (b) data in interval $-1 \mathrm{~T} \leq B \leq+1 \mathrm{~T}$.

1. Magnetization at $T=2 \mathrm{~K}$ and $H=9 \mathrm{~T}$ decreases from $\approx 0.1 \mu_{\mathrm{B}} /$ f.u. for the sample synthesized at $240{ }^{\circ} \mathrm{C}$ to 0.018 and $0.012 \mu_{\mathrm{B}} /$ f.u. for the samples synthesized at $600{ }^{\circ} \mathrm{C}$ and $1300^{\circ} \mathrm{C}$, respectively.

2. Hysteresis loop is observed for samples synthesized at temperatures up to $600^{\circ} \mathrm{C}$. The loop shape is characteristic of soft magnetic materials. The coercivity field and remanence decrease with increase of synthesis temperature and grain size (see the inset in Fig. 2 and Table II).

TABLE II

Magnetic parameters determined from the hysteresis loop: $\sigma$ - magnetization at $2 \mathrm{~K}$ and $9 \mathrm{~T}$; $\sigma_{\mathrm{r}}$ - remanence at $H=0 ; H_{\mathrm{c}}-$ coercivity field.

\begin{tabular}{c|c|c|c}
\hline \hline$T_{\mathrm{d}}\left[{ }^{\circ} \mathrm{C}\right]$ & $\sigma\left[\mathrm{emu} / \mathrm{g}_{\mathrm{NiO}}\right]$ & $\sigma_{\mathrm{r}}\left[\mathrm{emu} / \mathrm{g}_{\mathrm{NiO}}\right]$ & $B[\mathrm{~T}]$ \\
\hline 240 & 6.85 & 0.84 & 0.040 \\
290 & 6.63 & 0.75 & 0.038 \\
350 & 4.75 & 0.50 & 0.028 \\
400 & 4.65 & 0.38 & 0.023 \\
470 & 3.27 & 0.17 & 0.010 \\
600 & 1.33 & 0.07 & 0.008 \\
1300 & 0.92 & 0.02 &
\end{tabular}

\subsection{Neutron diffraction}

In purpose to explain the origin of low temperature anomaly a neutron diffraction experiment has been performed for two samples obtained at 240 and $1300^{\circ} \mathrm{C}$, respectively.

For the sample annealed at $1300^{\circ} \mathrm{C}$, scans in full $2 \theta$ range $\left(2 \theta=4^{\circ}-134^{\circ}\right)$ were collected at $2.6,24.5$ and $556 \mathrm{~K}$ (Fig. 5) together with scans in limited $2 \theta$ range at a number of temperatures between 2.6 and $534 \mathrm{~K}$. The neutron diffraction pattern taken at $556 \mathrm{~K}$ contains only the 111, 200 and 220 peaks of nuclear origin. In the patterns measured at 2.6 and $24.5 \mathrm{~K}$ additional peaks of magnetic origin corresponding to the $2 a \times 2 a \times 2 a$

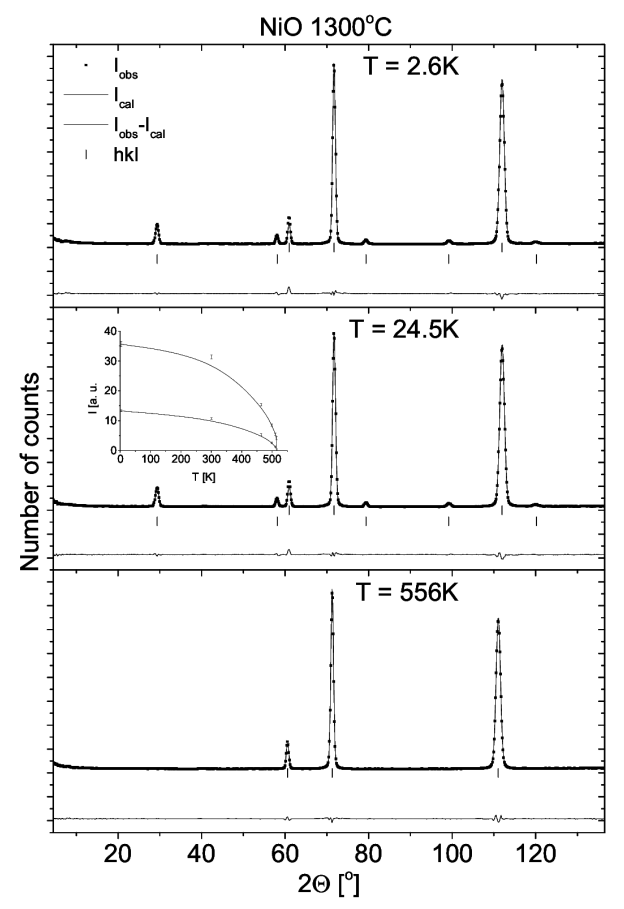

Fig. 5. Neutron diffraction patterns of $\mathrm{NiO}$ synthesized at $1300{ }^{\circ} \mathrm{C}$ measured at 2.6, 24.5 and $556 \mathrm{~K}$. The squares represent experimental points. The solid lines are the calculated profiles for crystal and magnetic structure models described in the text and the difference between observed and calculated intensities (at the bottom of diagram). The vertical bars indicate Bragg reflections of nuclear and magnetic origin. The inset show intensity vs. temperature dependence of the $\frac{1}{2}, \frac{1}{2}, \frac{1}{2}$ and $\frac{3}{2}, \frac{1}{2}, \frac{1}{2}$ magnetic peak.

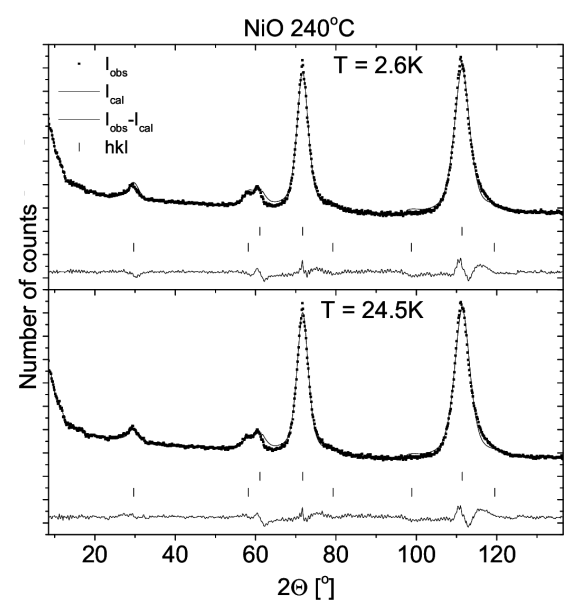

Fig. 6. Neutron diffraction patterns of the $\mathrm{NiO}$ sample synthesized at $240{ }^{\circ} \mathrm{C}$ collected at 2.6 and $24.5 \mathrm{~K}$. All other descriptions are the same as in Fig. 5.

magnetic unit cell are observed. Numerical analysis of the data lead to magnetic structure similar to that reported previously $[2-4]$. The determined magnetic moment of $\mathrm{Ni}^{2+}$ ion equals 2.16(4) $\mu_{\mathrm{B}}$ and 2.14(4) $\mu_{\mathrm{B}}$ at 2.6 and $24.5 \mathrm{~K}$, respectively. These values are close to those reported in Ref. [11]. Temperature dependence of 
the $\frac{1}{2}, \frac{1}{2}, \frac{1}{2}$ and $\frac{3}{2}, \frac{1}{2}, \frac{1}{2}$ magnetic peaks provides the Néel temperature equal to $516 \mathrm{~K}$ which is in agreement with the one reported in Ref. [2].

The Bragg reflections for the sample prepared at $240^{\circ} \mathrm{C}$ show significant broadening when compared with those found the sample obtained at $1300^{\circ} \mathrm{C}$ (see Fig. 6). The patterns measured at 2.6 and $24.5 \mathrm{~K}$ contain strong peaks of nuclear origin accompanied with weak reflec- tions of magnetic origin. The full width at half maximum (FWHM, denoted below by $H_{h k l}$ ) has been fitted using the formula

$$
\left(H_{h k l}\right)^{2}=U \tan ^{2} \theta+V \tan \theta+W
$$

where $U, V$, and $W$ are half-width parameters. All the data derived from neutron diffraction measurements are summarized in Table III.

TABLE III

Structural parameters from the neutron diffraction data: $T_{\mathrm{d}}$ - dehydration temperature, $T$ - temperature of measurement, $a$ - lattice parameter, $\mu-\mathrm{Ni}^{2+}$ magnetic moment, $U, V, W-$ half-width parameters.

\begin{tabular}{c|c|c|c|c|c|c|c|c}
\hline \hline$T_{\mathrm{d}}\left[{ }^{\circ} \mathrm{C}\right]$ & $T[\mathrm{~K}]$ & $a[\AA]$ & $\mu\left[\mu_{\mathrm{B}}\right]$ & $U$ & $V$ & $W$ & $R_{\mathrm{N}}[\%]$ & $R_{\mathrm{mag}}[\%]$ \\
\hline 1300 & 2.6 & $4.1741(2)$ & $2.16(4)$ & $1.18(7)$ & $-1.27(14)$ & $0.91(6)$ & 2.20 & 2.11 \\
& 24.5 & $4.1738(2)$ & $2.14(4)$ & $1.27(7)$ & $-1.45(15)$ & $1.04(7)$ & 1.82 & 1.65 \\
& 555.0 & $4.1959(3)$ & & $1.18(7)$ & $-1.25(14)$ & $0.89(6)$ & 0.40 & \\
\hline 240 & 2.6 & $4.2013(9)$ & $1.84(7)$ & $5.56(91)$ & $-1.40(45)$ & $7.60(85)$ & 2.20 & 5.6 \\
& 24.5 & $4.2003(9)$ & $1.82(7)$ & $6.00(95)$ & $-0.90(51)$ & $8.04(81)$ & 1.83 & 8.2
\end{tabular}

\section{Conclusions}

The work presents results for nanoparticle $\mathrm{NiO}$ samples obtained by thermal decomposition of $\mathrm{Ni}(\mathrm{OH})$ hydroxide. The X-ray diffraction data lead to conclusion that the lattice constant $a$ together with unit cell deformation parameter $\Delta a / a$ decreases while grain size $D$ increases with increasing decomposition temperature $T_{\mathrm{d}}$. Also the magnetic data indicate a change of physical properties due to $T_{\mathrm{d}}$ temperature: magnetic susceptibility, magnetization and hysteresis loop decrease with increase of $T_{\mathrm{d}}$ temperature.

A general conclusion that may drawn is the following: $\mathrm{NiO}$ samples obtained by thermal decomposition of the $\mathrm{Ni}(\mathrm{OH})_{2}$ hydroxide have cubic crystal structure of the NaCl-type. The grain size increases with increasing synthesis temperature $T_{\mathrm{d}}$. The grain size influences:

- crystal structure: both the lattice constant $a$ and crystal unit cell deformation parameter $\Delta a / a$ are affected;

- magnetic properties: hysteresis loop is observed only for samples with annealing tempeatures up to $600{ }^{\circ} \mathrm{C}$. Remanence and coercivity field decreases with increase of $T_{\mathrm{d}}$ temperature. Small values of magnetization together with appearance of hysteresis loop characteristic of ferro- or ferrimagnetic materials indicate existence of not fully compensated antiferromagnetism in the investigated samples;

- neutron diffraction data obtained for the sample annealed at $1300{ }^{\circ} \mathrm{C}$ are similar to those reported in Refs. [3-5]. The low temperature antiferromagnetic order corresponds to magnetic unit cell with doubled lattice constant in comparison with the crystal one. The neutron diffraction patterns of the sample synthesized at $240^{\circ} \mathrm{C}$ show significant broadening of both the Bragg reflection of nuclear and magnetic origin indicating drastic decrease of grain size.

In all investigated samples a maximum in $\mathrm{ZFC}$ curve is observed at low temperature region, close to $10 \mathrm{~K}$. The maximum decreases with increase of annealing temperature. In case of the FC curves a significant change of magnetic susceptibility is visible in the same temperature region.

ZFC curves of the samples annealed at $240^{\circ} \mathrm{C}$ and $290^{\circ} \mathrm{C}$ show a broad maximum at high temperatures. This maximum shifts towards higher temperatures for samples with higher annealing temperatures and disappears for the samples annealed at $600^{\circ} \mathrm{C}$ and $1300^{\circ} \mathrm{C}$. Such a result indicates that this maximum is strictly correlated with grain size. No maxima are observed for the FC curves at high temperature region.

Presented neutron diffraction data indicate no change of magnetic order at temperatures at which above mentioned maxima in magnetic susceptibility are observed, concerning both the low and high temperature region (Ref. [6] and Fig. 13 in Ref. [12]). Thus the maxima are rather related to some surface effects playing a growing role with decreasing particle size due to increase of the surface to volume ratio. According to the Néel model [13] antiferromagntic nanoparticles have a net magnetic moment resulting from uncompensated number of spins in two sublattices. The net moment depends on particle morphology. Existence of net magnetic moment in the investigated samples is confirmed by small values of magnetization and hysteresis loop behavior (see Fig. 4). Hence, observed at high temperatures broad maxima are associated with progressive blocking of nanoparticle moment, i.e. uncompensated moment of particle core, which takes place with decreasing temperature.

\section{Acknowledgments}

This research project has been supported by the European Commission under the 7th Framework Programme 
through the "Research Infrastructure" action of the "Capacities" Programme, NMI3-II Grant number 283883, the European Regional Development Fund in the framework of the Polish Innovation Economy Operational Program (contract no. POIG.02.01.00-12-023/08).

\section{References}

[1] J.L. Dormann, D. Fiorani, E. Tronc, Adv. Chem. Phys. 98, 283 (1997).

[2] M.F. Trombe, J. Phys. Radium 12, 170 (1951).

[3] C.G. Shull, W.A. Strauser, E.O. Wollan, Phys. Rev. 83, 333 (1951).

[4] W.L. Roth, Phys. Rev. 110, 1333 (1958) and Phys. Rev. 111, 772 (1958).

[5] W.L. Roth, G.A. Slack, J. Appl. Phys. 31, S525 (1960).

[6] J.T. Richardson, W.O. Milligan, Phys. Rev. 102, 1289 (1956).
[7] E. Winkler, R.D. Zysler, M. Vasquez Mansilla, D. Fiorani, D. Rinaldi, M. Vasilakaki, K.N. Trohidou, Nanotechnology 19,185702 (2008); E. Winkler, R.D. Zysler, M. Vasquez Mansilla, D. Fiorani, Phys. Rev. B 72, 132409 (2005).

[8] S.D. Tiwari, K.P. Rajeev, Phys. Rev. B 72, 104433 (2005).

[9] J. Rodriguez-Carvajal, Physica B 192, 55 (1993).

[10] G.K. Williamson, W.H. Hall, Acta Metall. 1, 22 (1953).

[11] M.T. Hutchings, E.J. Samuelsen, Phys. Rev. B 6, 3447 (1972).

[12] A. Szytuła, Solid State Phenom. 112, 39 (2006).

[13] L. Néel, Compt. Rend. Hebdomad. Séances Acad. Sci. 252, 4075 (1961); Compt. Rend. Hebdomad. Séances Acad. Sci. 253, 9 (1961); Compt. Rend. Hebdomad. Séances Acad. Sci. 253, 1286 (1961). 